\title{
Asset Trading Volume with Dynamically Complete Markets and Heterogeneous Agents*
}

\author{
Kenneth L. Judd \\ Hoover Institution \\ Stanford, CA 94305 \\ judd@hoover.stanford.edu
}

Felix Kubler

Dept. of Economics

Stanford University

Stanford, CA 94305

fkubler@leland.stanford.edu

\author{
Karl Schmedders \\ Kellogg Graduate School of Management \\ Northwestern University \\ Evanston, IL 60208 \\ k-schmedders@nwu.edu
}

May 1, 2000

*The authors are grateful to Larry Jones, Mordecai Kurz, Michael Magill, and Tom Sargent, and seminar participants at Carnegie Mellon University for helpful comments. 


\begin{abstract}
:
The trading volume of long-lived securities with recursive payoffs, such as equity, is generically zero in infinite-horizon recursive pure exchange Lucas asset models with heterogeneous agents. In equilibrium, there is no portfolio rebalancing of such assets. More generally, the end-of-period portfolio of long- and short-lived securities is constant over time and states in the generic economy. We also present a nonrobust formulation of dynamically complete markets which does have nonzero trading volume in equilibrium. The comparisons show that any theory of asset trading volume will be very sensitive to small changes in model specifications.
\end{abstract}




\section{Introduction}

The Lucas asset pricing model (Lucas (1978)) examines a representative agent endowment economy. From an asset-pricing point of view this assumption is sensible if markets are complete or can be completed through dynamic trading of the available securities (see Kreps (1982)). In this case, even if there are several agents in the economy, asset prices evolve as if there were a single agent. Therefore, asset pricing research typically assumes a representative agent. This approach ignores trading volume, an unfortunate feature since there is data on volume. Even when markets are complete, a recognition of possible agent heterogeneity might enrich the Lucas model by jointly modeling asset pricing and trading volume. This paper examines a simple extension of Lucas (1978) with agent heterogeneity and dynamically complete markets. We characterize equilibrium and present an algorithm to compute equilibrium prices and trading volume. We use these results to study how the set of available securities affects trading volume.

It is commonly thought that trading comes from a combination of differences in information, beliefs, and tastes. Many models assume that the most important source for trade is informational asymmetries among agents combined with other shocks (see e.g. Wang (1994)). Brock and LeBaron (1996) present an adaptive beliefs model which is able to roughly reproduce several features seen in the data.

Another presumed source of volume is portfolio rebalancing, i.e. individuals with common beliefs adjusting their portfolios in response to new public information about assets' future returns. If there is a complete set of Arrow securities then they are traded at the initial period and no further asset trading occurs. When there does not exist a complete set of Arrow securities, agents may need to periodically trade assets to implement their desired consumption plan. Therefore, one would expect there to be trade even with symmetric information and dynamically complete security markets. As Grossman (1985) puts it, "with incomplete equitization, but complete markets, there is trading on each day, as the person with a low income on that day enters the loan market to lend. A particular person will be a bond buyer on some days and a bond seller on the other."

The key result in this paper is that this intuition is misleading when asset markets are dynamically complete and the economy is stationary. In particular, we find that if all assets are long-lived, such as equity or consols, then there is no asset-trading after the initial period. The intuition is clear and follows directly from linear algebra. Suppose, for the sake of simplicity, that the current dividend summarizes all information about future dividends ${ }^{1}$. Then the dividend process is a Markov process where we identify the current state $^{2}$ of the Markov chain with the current dividend.

\footnotetext{
${ }^{1}$ This is not necessary. All that is needed is that the current state of the dividend process is common knowledge.

${ }^{2}$ We need to be careful to distinguish the two possible meanings of the term "state." In the
} 
Suppose that there are $S$ states and $S$ long-lived securities where each security's payoff depends solely on the current dividend. Assume further that the $S$ securities are linearly independent. If utility is separable over time with exponentially discounted utility (as assumed in Lucas and below) then each agent's optimal consumption policy is a function of the exogenous state, and is also a vector of $S$ numbers. If the state-contingent dividends from the $S$ long-lived assets are $S$ independent vectors, any state-contingent consumption plan equals the returns generated by some unique fixed and constant combination of the $S$ assets. If an agent's endowment does not equal the fixed portfolio which produces the desired consumption process, he can obtain that fixed portfolio through trading in the initial period. Therefore, any consumption plan can be implemented by some trade-once-and-hold-forever trading strategy. By concavity, there is a unique optimal consumption plan; hence, the trade-once-andhold-forever strategy which can implement the optimal consumption process must be the unique optimal trading strategy. This is true for each agent and for any price process. Therefore, it must hold in equilibrium.

This simple example assumed all assets were long-lived like equity. However, the essential logic continues to hold if there are some short-lived zero-net-supply securities. Our analysis allows the continual creation of single-period securities, such as short-term debt and options. In this case, agents will buy or sell the newly issued short-lived assets in each period. However, their portfolio of short-term securities will, in the appropriate sense, be the same at the end of each time in all states of the world. Suppose, for example, that an investor holds two shares, three 60-day call options, and one 30-day call option at the end of some period. Then we argue that he holds that portfolio at the end of any period, in any state and at any time. Of course, some trade is necessary as the character of the short-lived assets changes. Over the course of 30 days, the investor needs to sell two of those 60-day call options as they become 30-day options in order to maintain his position in 30-day options. Similar considerations apply to bonds with a finite maturity. Our general conjecture is that an investor will trade short-lived securities as they mature to maintain a constant maturity structure in his portfolio. We prove this for a limited set of securities in this paper, but present the general conjecture here to clarify the general intuition.

This argument depends on all the special features of the Lucas model. If we replace the representative agent feature with an overlapping generations structure then there will be trade for life-cycle reasons. If asset markets are incomplete there will be trade. The key result here is that, contrary to standard intuition, there is no trade purely motivated by portfolio rebalancing.

The generic no-trade result we prove for equity and other long-lived assets is sur-

Arrow security approach, the states differ in both the time and dividend dimensions. In a Markov chain, the term state typically refers only to the current state of information about the current and future value of the dividend. In this paper, we will mean the state of the Markov process describing dividends when we say "state" unless it is clear we mean otherwise. 
prising. We then present special, nongeneric cases of the generalized Lucas model with heterogeneous agents and asset markets that are formally not complete but can be completed by trade. In these cases equity trade occurs in equilibrium for conventional reasons. These cases assume that there are many possible states but that only a few states are reachable from any particular state. This fact corresponds to a sparse transition matrix for the Markov transition process. Sparseness will imply that dynamic trading in a small number of assets can implement arbitrary consumption processes. Trade occurs because the short-run spanning properties of the assets change over time and investors adjust their portfolios accordingly.

In order to examine these features of our asset-pricing model, we compute some examples. We develop an algorithm to compute equilibrium prices and trading volume in the Lucas model with heterogeneous agents. The computation of dynamic equilibrium is generally difficult in infinite horizon models with incomplete markets (see Judd et al. 1998). However, since the equilibrium is Pareto efficient, we can use a Negishi approach to compute equilibrium. The resulting algorithm is simple enough to be implemented in an Excel spreadsheet. We compute some representative examples of the model. Our sparse transition matrix examples do follow the conventional intuition that portfolio rebalancing will occur in equilibrium. However, even in these example, the pattern of trade does not follow any simple intuitive pattern. While these examples are formally not robust to changes in the transition matrix, many will find them to be more intuitive descriptions of real asset markets and more suitable models of portfolio rebalancing.

The model examined here is simple, but makes points relevant for any discussion of volume. The results presented below show that any results on volume will be quite sensitive to the structure of the dividend process and the kind of assets being traded. Therefore, volume information will be quite difficult to interpret unless one has very precise knowledge about these details.

The paper is organized as follows. Section 2 describes the standard model of an infinite-horizon pure exchange economy. The concepts of Arrow-Debreu equilibrium and financial market equilibrium are defined. In Section 3 we present the numerical procedure for computing equilibrium, show that end-of-period asset holdings are generically constant, and illustrate the main points with an example. Section 4 presents examples of economies with sparse transition matrices with trading. Section 5 concludes.

\section{The Asset Market Economy}

We examine a standard Lucas asset pricing model with heterogeneous agents and complete asset markets. Time is indexed by $t \in \mathbb{N}_{0} \equiv\{0,1,2, \ldots\}$. A time-homogeneous Markov process of exogenous states $\left(y_{t}\right)_{t \in \mathbb{N}_{0}}$ is valued in a discrete set $Y=\{1,2, \ldots, S\}$. 
The Markov transition matrix is denoted by $\Pi$. A date-event $\sigma_{t}$ is the history of states up to time $t$, i.e. $\sigma_{t}=\left(y_{0}, y_{1}, \ldots y_{t}\right){ }^{3}$ Let $\Sigma_{t}$ denote the possible histories $\sigma_{t}$ up to time $t$. Let $\sigma \in R^{\infty}$ denote a complete history. Let $\Sigma=\cup_{t} \Sigma_{t}$ denote all possible histories of the exogenous states.

We assume a finite number of types $\mathcal{H}=\{1,2, . ., H\}$ of infinitely-lived agents. There is a single perishable consumption good, some of it produced by the firms and some if it being endowed to individuals. The firms distribute their output each period to its owners through dividends. investors trade in securities in order to transfer wealth across time and states. We assume that markets are complete with $J=S$ linearly independent assets traded on financial markets. Without loss of generality and for ease of notation we assume that each asset is either an infinitely-lived (longlived) asset or a single-period asset. Equity is an example of a long-lived asset, as is any asset with payments tied to the dividend process. Options and short-term debt are examples of the single-period assets. We will also assume that the short-lived assets are in zero net supply.

We assume that there are $J^{\ell} \geq 0$ long-lived assets. Asset $j$ pays a dividend $d^{j}: Y \rightarrow \mathbb{R}_{+}, j=1, \ldots, J^{\ell}$, which depends solely on the current state $y \in Y$. In addition, there are $J^{s}=S-J^{\ell} \geq 0$ short-lived securities issued in each period. Short-lived asset $j$ issued in period $t$ pays $d^{j}: Y \rightarrow \mathbb{R}_{+}, j=J^{\ell}+1, \ldots, S$, in period $t+1$, and then expires. We denote agent $h$ 's portfolio at time $t$ along $\sigma$ by $\theta_{t}^{h}(\sigma)=$ $\left(\theta_{t}^{h 1}(\sigma), \ldots, \theta_{t}^{h S}(\sigma)\right)=\left(\theta_{t}^{h l}(\sigma), \theta_{t}^{h s}(\sigma)\right) \in \mathbb{R}^{S}$, where $\theta_{t}^{h l}(\sigma) \in \mathbb{R}^{J^{\ell}}\left(\theta_{t}^{h s}(\sigma) \in \mathbb{R}^{J^{s}}\right)$ denotes agent $h$ 's portfolio of long-lived (short-lived) assets at time $t$ along $\sigma \in \Sigma$. His initial endowment of the long-lived assets prior to time 0 is denoted by $\theta_{-1}^{h l}$. We assume that the agent has zero initial endowment of the short-lived assets and, in order to rule out speculative bubbles, that all infinitely-lived assets are in positive net supply. agent $h$ also has an individual endowment of the consumption good at each time. This represents other sources of income, such as labor, and is a function $e^{h}: Y \rightarrow \mathbb{R}_{++}$depending on the current state $y_{t}$ alone. The aggregate endowment of the economy in state $y$ is $e(y)=\sum_{h=1}^{H}\left(e^{h}(y)+\theta_{-1}^{h l} d^{l}(y)\right)$.

Each agent $h$ has a time-separable state-dependent utility function

$$
U_{h}(c)=E\left\{\sum_{t=0}^{\infty} \beta^{t} u_{h}\left(c_{t}, y_{t}\right)\right\}
$$

where $c$ is a consumption process. We assume that the state-dependent utility functions $u_{h}(., y): \mathbb{R}_{++} \rightarrow \mathbb{R}$ are strictly monotone, $C^{2}$, strictly concave, and satisfy the Inada property, that is, $\lim _{c \rightarrow 0} u^{\prime}(c, y)=\infty$ where we let $u^{\prime}(c, y)$ denote $\frac{\partial}{\partial c}(u(c, y))$. We assume that the discount factor $\beta \in(0,1)$ is the same for all agents, and that all

\footnotetext{
"In this paper, we use the term "state" to refer to the state of the Markov process which describes dividends and other income. The term "date-event" refers to what is called a "contingent state" in Arrow (1959) and "event" in Debreu (1959).
} 
agents agree ${ }^{4}$ on the transition matrix for the dividend process.

Note that this representation of agents' preferences is a generalization of vonNeumann-Morgenstern utility in the sense that we allow each agent's utility function to depend on the exogenous state as well as consumption. The key is that we assume that the shocks to preferences lie in the span of the securities. Otherwise, there would be trading for hedging purposes. Since we want to focus on the role of portfolio rebalancing motives for trading, we abstract from hedging motivations.

Let the matrices

$$
e=\left(\begin{array}{ccc}
e^{1}(1) & \cdots & e^{1}(S) \\
\vdots & & \vdots \\
e^{H}(1) & \cdots & e^{H}(S)
\end{array}\right), \quad d=\left(\begin{array}{ccc}
d^{1}(1) & \cdots & d^{1}(S) \\
\vdots & & \vdots \\
d^{S}(1) & \cdots & d^{S}(S)
\end{array}\right)
$$

represent individual endowments and security dividends. The vector of utility functions is $u=\left(u^{1}, \ldots, u^{H}\right)$. We collect the primitives of the economy with financial markets in the expression $\mathcal{E}=(e, d, \Pi, \beta, u)$.

This is a simple model but includes many features thought to affect trading volume. For example, we include income shocks to individuals, a factor which Grossman(1985) argues to be important. We also allow individuals to have different tastes for risk and for the dividend process to have time-varying mean and variance. The presence of these factors makes it a reasonable model to use to study trading volume with heterogeneous agents.

\subsection{Arrow-Debreu Equilibrium}

Let $\omega^{h}(y)=e^{h}(y)+\theta_{-1}^{h \ell} d^{\ell}(y)$ denote the initial endowment of agent $h \in \mathcal{H}$ in state $y$. For an economy $\mathcal{E}$, define an Arrow-Debreu equilibrium which would be obtained in a world with an Arrow contingent security for every date-event. We take consumption at time 0 to be the numeraire. The price $p_{t}$ of the consumption good at $\sigma_{t}$ is denoted $p_{t}(\sigma)$; let $p(\sigma)$ denote the collection of prices. Similarly for the consumption plan $c_{t}(\sigma)$ and the consumption plan $c(\sigma)$.

Definition 1 An Arrow-Debreu equilibrium for an economy $\mathcal{E}$ is a collection of prices $(\bar{p}(\sigma))_{\sigma \in \Sigma}$ and consumption plans $(\bar{c}(\sigma))_{\sigma \in \Sigma}$ satisfying following conditions:

(1) $\sum_{h=1}^{H} \bar{c}_{t}^{h}(\sigma)=\sum_{h=1}^{H} \omega^{h}\left(y_{t}\right) \quad$ for all $\sigma \in \Sigma$ and all $t$

(2) For each agent $h, \bar{c}^{h}(\sigma)$ maximizes $U_{h}\left(c^{h}\right)$ given the lifetime budget constraint $\sum_{\sigma \in \Sigma} \sum_{t} \bar{p}_{t}(\sigma) \omega_{t}^{h}(\sigma)=\sum_{\sigma \in \Sigma} \sum_{t} \bar{p}_{t}(\sigma) c_{t}^{h}(\sigma)$.

\footnotetext{
${ }^{4}$ Conventional rational expectations assume that the agents know the true transition probabilities. The only thing we need for our analysis is that agents agree.
} 
Bewley (1972) proved that the economy $\mathcal{E}$ has an Arrow-Debreu equilibrium. The first and the second welfare theorem also hold. These facts allow us to analyze the dynamic stochastic equilibrium.

\subsection{Financial Markets Equilibrium}

It is unreasonable to assume that there is a separate security for each time and state contingency. Instead, the Lucas model and our generalization forces agents to use the $J$ available assets to achieve the desired consumption stream. At each state $y_{t}$ and along each history $\sigma$ each agent faces the budget constraint

$$
c_{t}^{h}(\sigma)=e^{h}\left(y_{t}\right)+\theta_{t-1}^{h s}(\sigma) d^{s}\left(y_{t}\right)+\theta_{t-1}^{h l}(\sigma)\left(q_{t}^{l}(\sigma)+d^{l}\left(y_{t}\right)\right)-\theta_{t}^{h}(\sigma) q_{t}(\sigma),
$$

where $d^{l}\left(y_{t}\right)\left(d^{s}\left(y_{t}\right)\right)$ equals the column vector of the payoffs of the long-lived (shortlived) assets given the state $y_{t}$.

The notion of a financial market equilibrium is defined as follows.

Definition 2 A financial markets equilibrium for an economy $\mathcal{E}$ is a process of portfolio holdings $\left\{\left(\bar{\theta}^{1}, \ldots, \bar{\theta}^{H}\right)\right\}$ and asset prices $\left\{\left(\bar{q}^{1}, \ldots, \bar{q}^{J}\right)\right\}$ satisfying the following conditions:

(1) $\sum_{h=1}^{H} \bar{\theta}_{t}^{h}(\sigma)=\sum_{h=1}^{H} \theta_{-1}^{h}$ for all $\sigma \in \Sigma, t>0$.

(2) For each agent $h$ :

$$
\begin{aligned}
\bar{\theta}^{h}(\sigma) & \in \arg \max _{\theta, c} U_{h}(c) \\
c_{t}^{h}(\sigma) & =e^{h}\left(y_{t}\right)+\bar{\theta}_{t-1}^{h s}(\sigma) d^{s}\left(y_{t}\right)+\bar{\theta}_{t-1}^{h l}(\sigma)\left(\bar{q}_{t}^{l}+d^{l}\left(y_{t}\right)\right)-\bar{\theta}_{t}^{h}(\sigma) \bar{q}_{t}(\sigma) \\
\sup _{\sigma \in \Sigma, t}\left|q_{t}(\sigma) \bar{\theta}_{t}^{h}(\sigma)\right| & <\infty
\end{aligned}
$$

It is well-known that in the absence of speculative bubbles, if there are as many assets as states then there is generically a one-to-one correspondence between ArrowDebreu equilibria and financial market equilibria; we will make this point precise below.

\section{A Theorem on Constant Portfolios}

In this section we show that for a generic set of short-lived assets' dividends every contingent markets equilibrium is equivalent to a financial markets equilibrium in which the end-of-period portfolio holdings of all agents are constant after an initial adjustment in period 0 . We do this in a constructive manner by describing an algorithm for the computation of equilibria in our model. 
We proceed in three steps. First, the welfare theorems tell us that the equilibrium allocation can be obtained as the solution of a representative agent's maximization problem over $h$ consumption goods. The artificial representative agent has a separable utility function

$$
E\left\{\sum_{h=1}^{H} \lambda^{h} \sum_{t=0}^{\infty} \beta^{t} u_{h}\left(c_{t}, y_{t}\right)\right\}
$$

where the $\lambda^{h}$ are the Negishi-weights. We compute the Negishi weights $\lambda^{h}, h=$ $2, \ldots, H$, in order to obtain a Pareto efficient sharing rule. This will allow us to determine the security prices $\bar{q}$ and derive the optimal portfolio choices $\bar{\theta}^{h}$ for all agents $h=1, \ldots, H$. In fact, once we have computed a Pareto efficient consumption allocation we will be able to give closed-form solutions for both asset prices and portfolios.

\subsection{Equilibrium Computation}

The following theorem is the basis for showing that all relevant economic variables exhibit time-homogeneity. It follows directly from the first welfare theorem.

Theorem 1 For an economy $\mathcal{E}$ every Arrow-Debreu equilibrium exhibits time-homogeneous Markovian consumption processes for all agents.

Proof: Denote the period 0 probability of event $\sigma_{t}$ by $\pi\left(\sigma_{t}\right)$. Suppose that there is an equilibrium where for two date-event nodes $\sigma_{t}, \sigma_{t^{\prime}}$ with $y_{t}=y_{t^{\prime}}$ we have $c_{t}^{h^{\prime}}(\sigma) \neq c_{t^{\prime}}^{h^{\prime}}(\sigma)$ for some agent $h \in H$. Then we could improve everybody's utility by redistributing consumption at these nodes. Let

$$
c_{t}^{h}(\sigma)=\frac{\beta^{t} \pi_{t}(\sigma) c_{t}^{h}(\sigma)+\beta^{t^{\prime}} \pi_{t^{\prime}}(\sigma) c_{t^{\prime}}^{h}(\sigma)}{\beta^{t} \pi_{t}(\sigma)+\beta^{t^{\prime}} \pi_{t^{\prime}}(\sigma)}
$$

for all $h \in H$. This convex combination is clearly a feasible allocation and by strict concavity agent $h^{\prime}$ will derive higher utility. Therefore, $c^{h^{\prime}}\left(\sigma_{t}\right) \neq c^{h^{\prime}}\left(\sigma_{s}\right)$ contradicts efficiency.

The recursive property of equilibrium proven in Theorem 1 is the key to all of our results. First, note that the artificial representative agent's utility function can be rewritten as

$$
E\left\{\sum_{t=0}^{\infty} \beta^{t}\left(\sum_{h=1}^{H} \lambda^{h} u_{h}\left(c_{t}, y_{t}\right)\right)\right\}
$$

where the $\lambda^{h}$ are state- and time- independent weights on individual utilities.

Second, we take advantage of recursivity in our notation. We change the notation and express the dependence of all variables on the exogenous state through a subscript. 
The notation here will be inconsistent with the notation above, but will allow us to exploit the recursive nature of any equilibrium. For example, $c_{y}^{h}$ will denote the consumption of agent $h$ in state $y$.

We introduce some other useful notation. The state in a current period is denoted by $y$ and the random variable of the subsequent state is denoted by $y_{+}$. We will use a circle, o, to denote elementwise multiplication of vectors. Specifically, if $x, y \in R^{S}$ then

$$
x \circ y=\left(\begin{array}{c}
x_{1} y_{1} \\
x_{2} y_{2} \\
\vdots \\
x_{S} y_{S}
\end{array}\right) \in R^{S}
$$

Finally, $I_{S}$ is the $S \times S$ identity matrix.

\subsubsection{Step 1: Computing Negishi weights.}

A three-step process will compute the Negishi weights, individual consumption processes, asset prices, and individual portfolios. The first step is to compute the Negishi weights. At the optimal solution to the representative agent's optimization problem the derivatives $u_{h}^{\prime}\left(c_{y}^{h}, y\right)$ at each state $y$ are collinear across agents; that is,

$$
u_{1}^{\prime}\left(c_{y}^{1}, y\right)=\lambda^{h} u_{h}^{\prime}\left(c_{y}^{h}, y\right), h=2, \ldots, H .
$$

Since marginal utilities are collinear, we can replace Arrow-Debreu prices with the marginal utility of agent 1 . Therefore, we define

$$
p_{y}=u_{1}^{\prime}\left(c_{y}^{1}, y\right)
$$

to be the price of consumption in state $y$, and we let $p=\left(p_{y}\right)_{y \in Y} \in R^{S}$ be the vector of prices.

The budget constraint for each agent $h$ states that the present value of his consumption must equal the present value of his initial endowment. Let $V_{y}^{h}$ be the present value of consumption for agent $h$ when the economy starts in state $y \in Y$. We can compute $V_{y}^{h}$ by solving the recursive equation

$$
V_{y}^{h}=p_{y} c_{y}^{h}+\beta E\left\{V_{y_{+}}^{h} \mid y\right\}, y \in Y
$$

In matrix terms, (2) implies

$$
V^{h}=p \circ c^{h}+\beta \Pi V^{h}
$$

and has the unique solution

$$
V^{h}=\left[I_{S}-\beta \Pi\right]^{-1}\left(p \circ c^{h}\right)
$$


Let $W_{y}^{h}$ denote the present value of agent $h$ 's endowments and portfolio dividends; $W_{y}^{h}$ is the solution to

$$
W_{y}^{h}=p_{y} \omega_{y}^{h}+\beta E\left\{W_{y_{+}}^{h} \mid y\right\}, y \in Y
$$

The unique solution to (3) is

$$
W^{h}=\left[I_{S}-\beta \Pi\right]^{-1}\left(p \circ \omega^{h}\right) .
$$

If the economy starts in the state $y_{0} \in Y$ at period $t=0$ then the budget constraint for the Arrow-Debreu model requires that

$$
V_{y_{0}}^{h}=W_{y_{0}}^{h}, h=1, \ldots, H .
$$

Due to Walras' law it actually suffices to require this last equation for the first $H-1$ agents only. So, we require

$$
\left(\left[I_{S}-\beta \Pi\right]^{-1}\left(p \circ\left(c^{h}-\omega^{h}\right)\right)\right)_{y_{0}}=0 .
$$

for $h=1, \ldots, H-1$. Market clearing requires that

$$
\sum_{h=1}^{H} c_{y}^{h}=\sum_{h=1}^{H} \omega_{y}^{h} .
$$

The system of equations $(1,4,5)$ has the $H S+(H-1)$ unknowns

$$
\begin{array}{ll}
c_{y}^{h}, & y \in Y, h=1, \ldots, H, \\
\lambda^{h}, & h=2, \ldots, H .
\end{array}
$$

The system $(1,4,5)$ of nonlinear equations has as many equations as unknowns. The second welfare theorem implies that this system always has at least one solution. Any solution to $(1,4,5)$ is an equilibrium state-contingent consumption $c_{y}^{h}$ for agent $h=1, \ldots, H$ in state $y \in Y$.

\subsubsection{Step 2: Computing asset prices.}

Using the Euler equations of the first agent we can compute the price function of any asset. For a long-lived asset $j$ the Euler equations for agent 1 are

$$
q_{y}^{j} p_{y}=\beta E\left\{p_{y_{+}}\left(q_{y_{+}}^{j}+d_{y_{+}}^{j}\right) \mid y\right\}, y \in Y
$$

which is a system of $S$ linear equations in $S$ unknowns. The solution is

$$
q^{j} \circ p=\left[I_{S}-\beta \Pi\right]^{-1} \beta \Pi\left(p \circ d^{j}\right) .
$$


For a short-lived asset $j$ the Euler equations for agent 1 are

$$
q_{y}^{j} p_{y}=\beta E\left\{p_{y_{+}} d_{y_{+}}^{j} \mid y\right\}
$$

Therefore, the price for short-lived asset $j$ in state $y$ is

$$
q_{y}^{j}=\frac{\beta E\left\{p \circ d_{y+}^{j} \mid y\right\}}{p_{y}}=\frac{\beta \Pi_{y}\left(p \circ d^{j}\right)}{p_{y}} .
$$

\subsubsection{Step 3: Computing portfolios.}

We next examine the portfolios of the agents. They must also be recursive, that is, depending solely on the state $y$ and any state to which the dividend process can move from $y$. Assume that the transition matrix $\Pi$ has no zero elements; therefore, all states can be reached in one transition from each state. Then there is a budget constraint which must hold between any two dividend states stating that if the current state is $y$ and the previous state was $z$ then the end-of-period portfolio in state $z$ must finance the consumption and investment choices in state $y$. This collection of budget constraints for agent $h$ across the various states implies

$$
\theta_{z}^{h \ell}\left(q_{y}^{\ell}+d_{y}^{\ell}\right)+\theta_{z}^{h s}\left(d_{y}^{s}\right)=c_{y}^{h}-e_{y}^{h}+\theta_{y}^{h} q_{y} \quad \forall y, z \in Y
$$

a collection of $S^{2}$ equations for the $S^{2}$ unknown end-of-period portfolios. The key fact is that the right-hand side of (6) is the allocation of wealth across consumption and investment in state $y$ and, because of recursivity, cannot depend on $z$ whereas the left-hand side of (6) depends strongly on the previous period's state. Recursivity implies that an agent must have the proper resources in state $y$ to carry out the stationary plan for current and future consumption no matter what the state was in the previous period even though the initial wealth in state $y$ depends on the portfolio at the end of the previous period.. This clearly puts strong constraints on the possible values of $\theta_{z}^{h}$, the end-of-period portfolio of type $h$ agents in state $z$. In fact, we will see that, generically, this is possible only if $\theta_{z}^{h}$ is independent of the state $z$. We now present the details.

The system (6) is equivalent to:

$$
\begin{array}{lc}
\theta_{z}^{h \ell}\left(q_{s}^{\ell}+d_{s}^{\ell}\right)+\theta_{z}^{h s}\left(d_{s}^{s}\right)=\theta_{y}^{h \ell}\left(q_{s}^{\ell}+d_{s}^{\ell}\right)+\theta_{y}^{h s}\left(d_{s}^{s}\right) & y, z, s \in Y, z, y \neq s \\
\theta_{y}^{h \ell}\left(q_{y}^{\ell}+d_{y}^{\ell}\right)+\theta_{y}^{h s}\left(d_{y}^{s}\right)=c_{y}^{h}-e_{y}^{h}+\theta_{y}^{h} q_{y} & y \in Y
\end{array}
$$


which in turn is equivalent to:

$$
\begin{gathered}
\left(\theta_{y}^{h \ell}-\theta_{z}^{h \ell}, \theta_{y}^{h s}-\theta_{z}^{h s}\right)\left[\begin{array}{c}
q^{1}+d^{1} \\
\vdots \\
q^{J^{\ell}}+d^{J^{\ell}} \\
d^{J^{\ell}+1} \\
\vdots \\
d^{S}
\end{array}\right]=0, \quad \forall y, z \in Y \\
\theta_{y}^{h \ell} d_{y}^{\ell}+\theta_{y}^{h s}\left(d_{y}^{s}-q_{y}^{s}\right)=c_{y}^{h}-e_{y}^{h}, \quad \forall y \in Y
\end{gathered}
$$

where the row vector $q^{j}=\left(q_{1}^{j}, \ldots, q_{S}^{j}\right)$ denotes prices of asset $j$ across all states $y \in Y$. Similarly, $d^{j}=\left(d_{1}^{j}, \ldots, d_{S}^{j}\right)$ denotes the row vector of dividends of asset $j$ across all states. At this point in the argument we need that the $S \times S$-matrix

$$
\left[\begin{array}{c}
q^{1}+d^{1} \\
\vdots \\
q^{J^{l}}+d^{J^{l}} \\
d^{J^{l}+1} \\
\vdots \\
d^{S}
\end{array}\right]
$$

has full rank $S$. Kreps (1982) shows that the matrix in (9) has full rank for a generic set of asset dividends when agents have no initial holdings of the assets. His argument, combined with the fact that the long-lived assets' dividends are independent, shows that the matrix in (9) has full rank generically in the dividends of the short-lived assets. Then the equations (8) imply that

$$
\theta_{y}^{h}=\theta_{z}^{h}, \forall y, z \in Y
$$

Hence, we can define the state-independent portfolio vector $\Theta^{h} \equiv \theta_{y}^{h}$ for all $y \in Y$ and the equations (8) become

$$
\left(\Theta^{h \ell}, \Theta^{h s}\right)\left[\begin{array}{c}
d^{1} \\
\vdots \\
d^{J^{\ell}} \\
d^{J^{\ell}+1}-q^{J^{\ell}+1} \\
\vdots \\
d^{S}-q^{S}
\end{array}\right]=c^{h}-e^{h} .
$$


The $S \times S$-matrix

$$
\left[\begin{array}{c}
d^{1} \\
\vdots \\
d^{J^{\ell}} \\
d^{J^{\ell}+1}-q^{J^{\ell}+1} \\
\vdots \\
d^{S}-q^{S}
\end{array}\right]
$$

has full rank $S$ if and only if the matrix in equation (8) has full rank. So, for generic dividends of the short-lived assets the last system (10) implies that for all $h=1, \ldots, H$

$$
\left(\Theta^{h \ell}, \Theta^{h s}\right)=\left(c^{h}-e^{h}\right)\left[\begin{array}{c}
d^{1} \\
\vdots \\
d^{J^{\ell}} \\
d^{J^{\ell}+1}-q^{J^{\ell}+1} \\
\vdots \\
d^{S}-q^{S}
\end{array}\right]^{-1} .
$$

Substituting the equilibrium prices $\bar{q}$ into equation (11) leads to the equilibrium portfolio holdings $\bar{\Theta}^{h}$ for all agents $h=1, \ldots, H$.

Furthermore, there is a special case which deserves attention. Suppose that there are no short-lived assets and that the dividend payoff matrix $d$ is nonsingular. Being nonsingular is a generic property, so its assumption is natural. The absence of shortlived assets implies that asset prices $q$ do not appear in (11). Then, the nonsingularity of $d$ implies that (11) is surely solvable. This is the key special case which captures the basic intuition of the result.

Note that our derivation does not use the fact that the agents choose their consumption policies optimally. Any consumption allocation among the agents that is a first-order Markovian process and that satisfies market-clearing can be supported through constant portfolios. Put differently, for any set of welfare weights portfolios will be constant as long as markets clear. In addition, the portfolio choices can be expressed independently of the prices of the long-lived securities.

We summarize our findings for the equilibrium portfolios in the following theorem.

Theorem 2 Suppose that an infinite-horizon pure exchange economy has a timehomogeneous discrete-time and finite-state Markov dividend process. Furthermore, assume that the dividend process for the infinitely-lived assets has a Markov transition matrix $\Pi$ with no zero probabilities and the dividend payoffs of the infinitely-lived assets are linearly independent. Then the following holds: for a generic set of dividends of the short-lived assets the end-of-period portfolios of all agents are constant after one initial round of trading. Therefore, the trading volume for infinitely-lived 
securities is zero after the initial round of trading. Furthermore, if there are no shortlived assets then portfolios of all agents are constant after the initial period.

Theorem 2 shows that there will be no trade in assets after an initial period. This is a strong result which relies on many assumptions. We assume infinite-lived agents with additively separable utility and a common discount factor. An overlapping generations model will produce trade as may models with nonseparable preferences. The result is still surprising since we may think that heterogeneous risk preferences would motivate some portfolio rebalancing in equilibrium even in this simple model.

\subsection{Complete Markets with S - 1 Short-lived Assets}

For infinite-horizon economies with only short-lived assets we can use Theorem 2 in order to obtain another surprising result about equilibrium portfolios.

Theorem 3 Consider an infinite-horizon pure exchange economy with a time-homogeneous discrete Markov process of exogenous states and $S-1$ short-lived assets that have zero payoffs in the state $y_{0}$ in which the economy starts at time $t=0$. For a generic set of dividends of the short-lived assets the financial markets equilibrium is equivalent to the Arrow-Debreu equilibrium. Put differently, markets are complete with $S-1$ securities.

Proof: Consider an infinite-horizon pure exchange economy with a time-homogeneous discrete Markov process of exogenous states and $S$ short-lived assets. Theorem $2 \mathrm{im}-$ plies that in equilibrium we can write an agent's budget constraint at time $t=0$ as follows:

$$
c_{y_{0}}^{h}=e_{y_{0}}^{h}-\bar{\Theta}^{h} \bar{q}, \forall h .
$$

The theorem also implies that in equilibrium we can write an agent's budget constraint in state $y_{0}$ at any time $t>0$ as

$$
c_{y_{0}}^{h}=e_{y_{0}}^{h}+\bar{\Theta}^{h} d_{y_{0}}-\bar{\Theta}^{h} \bar{q}, \forall h .
$$

These two equations imply that

$$
\bar{\Theta}^{h} d_{y_{0}}=0, \forall h .
$$

Now suppose that there is only a single asset with nonzero payoffs in the state $y_{0}$. In that case the last equation implies that every agent has a zero position in that asset in all states. In other words, the agents only trade the other $S-1$ short-lived assets and still obtain complete markets consumption. This observation completes the proof of the theorem. 


\subsection{A Numerical Example}

The three steps of our algorithm are easy to implement. The first step, computing the Negishi weights and consumption functions, requires solving a nonlinear system of equations. The second and third step, computing the asset prices and the portfolios, respectively, require us only to solve linear systems of equations. For small examples it is possible to implement the algorithm in an Excel spreadsheet utilizing the solver add-in. In all the numerical examples of this and the next section the first system never required more than a few seconds to solve and the (relative) numerical errors ${ }^{5}$ were less than $10^{-10}$. The linear system of the second and third step were solved in less than a second with numerical errors less than $10^{-16}$. All computations were done on a 450Mhz PentiumPC using Excel with Windows97.

We illustrate the method with a simple example. Assume $H=2$ agents with CRRA Bernoulli-functions. Type 1 agents will be relatively risk tolerant with a coefficient of relative risk aversion of $\gamma_{1}=0.5$ and type 2 investors will have relative risk aversion of $\gamma_{2}=4$. The common discount factor equals $\beta=0.95$. Assume $S=3$ exogenous states. The economy starts in state 1 . The first asset is long-lived (call it stock) and has a dividend vector $d^{s}=(1,10,100)^{T}$. The stock is in unit net supply and the agents have both an initial endowment of $\theta_{-1}^{1 s}=\theta_{-1}^{2 s}=0.5$. Agents have no individual endowment of the consumption good, so $e_{y}^{1}=e_{y}^{2}=0$ for $y \in\{1,2,3\}$. The Markov transition matrix is

$$
\Pi=\left[\begin{array}{lll}
0.45 & 0.10 & 0.45 \\
0.05 & 0.90 & 0.05 \\
0.45 & 0.10 & 0.45
\end{array}\right] .
$$

The second asset is a riskless short-lived bond paying 1 unit of the consumption good in every state; so, $d_{y}^{b}=1$ for $y \in\{1,2,3\}$. The third asset is a one-period option on the stock with a strike price $K$ resulting in payoffs $d_{y}^{o}=\max \left\{q_{y}^{s}+d_{y}^{s}-K, 0\right\}, y \in$ $\{1,2,3\}$. Both the bond and the option are in zero net supply and the agents have zero endowments of these securities.

We first compute the Negishi weights. Since there are only two agents we need to compute only one Negishi weight, that of the second agent. Solving the optimality and feasibility conditions shows that $\lambda^{2}=14.86716$ and that state-contingent

\footnotetext{
5 "Relative numerical error" refers to the consumption equivalent error in the Euler equations. For example, when we say that we had an error less than $\varepsilon$ in solving an equation of the form $u_{1}^{\prime}\left(c_{y}^{1}, y\right)=\lambda^{h} u_{h}^{\prime}\left(c_{y}^{h}, y\right)$, we mean that our solutions satisfied

$$
\left|\frac{u_{1}^{\prime}\left(c_{y}^{1}, y\right)-\lambda^{h} u_{h}^{\prime}\left(c_{y}^{h}, y\right)}{u_{1}^{\prime}\left(c_{y}^{1}, y\right)}\right|<\varepsilon
$$
}


consumption is

$$
\begin{aligned}
& c^{1}=(0.00437,7.475,96.523) \\
& c^{2}=(0.9956,2.525,3.476)
\end{aligned}
$$

The solution for consumption allows us to compute asset prices for various assets. The Euler equations of the first agent imply that the prices of the stock and the bond satisfy

$$
\begin{aligned}
& q^{s}=(11.18,384.73,1662.39) \\
& q^{b}=(0.4327,2.833,64.315)
\end{aligned}
$$

These prices hold no matter what other asset is used to complete the market.

We can use the consumption solution to price any option which completes the market span. Not all options are acceptable. In order for markets to be complete, the strike price of the option must satisfy

$$
12.184<K<1762.3 \text {. }
$$

If $K<12.184$ then the option has positive payoffs in all states and is spanned by the payoffs of the stock and the bond. If $K>1762.3$ then the option payoffs are identically zero.

The option value will depend on the strike price. If $K=100$, the payoff vector of the option equals $d^{o}=(0,294.7267,1662.388)$. Following Step 2 of our algorithm the Euler equations yield the vector of option prices

$$
q^{o}=(5.458,273.97,811.28)
$$

Step 3 of the algorithm tells us that the constant portfolio for the first agent is

$$
\Theta^{1}=\left(\theta^{1 s}, \theta^{1 b}, \theta^{1 o}\right)=(0.5940,-2.7198,-0.1185)
$$

and the constant portfolio of agent 2 is

$$
\Theta^{2}=(0.405965,2.71981,0.11854)
$$

For the case of $K=1000$, a much higher strike price, $d^{o}=(0,0,762.388)$. The option has a nonzero payoff in only one of the three states. In this case the option price vector equals

$$
q^{o}=(2.192614,10.07765,325.921) .
$$

and the agents' end-of-period portfolios are

$$
\begin{aligned}
& \Theta^{1}=(0.502703,-1.06706,-0.04882) \\
& \Theta^{2}=(0.497297,1.06706,0.04882) .
\end{aligned}
$$


in each state. In this case, type one agents hold less equity and sell fewer options. We see that the strike price of the option affects the equilibrium portfolio since it affects the covariance patterns among the assets. The assets span the same space but different combinations are used to implement any specific consumption plan. However, the portfolio is constant with no trade in the equity and bond markets.

\section{Dynamically Complete Markets with Few Assets}

The results obtained above are initially unintuitive. Standard intuition says that investors should continuously trade securities in response to new information about expected future returns and their riskiness. A critical feature of our result is that it still assumes a large set of securities. For generic processes involving $S$ states, we will need all of the $S$ assets specified above. It is more natural to assume that real markets contain fewer assets than the number of possible states. We could examine the impact of incomplete markets on asset volume; Judd et al. (1999, 2000) take such an approach to models with one and two assets. In this paper, we want to stay with the complete market framework and stay away from the efficiency issues which arise in any model with genuine asset incompleteness.

In this section, we examine some examples where trading in a few assets can implement Pareto efficient dynamic consumption processes. From a mathematical point of view, almost all transition matrices $\Pi$ will have no zero entries, implying that each state can be followed by any other state. In this case, completeness of markets require $S$ assets. However, some may find it more intuitive to believe that dividends move more "smoothly" and do not display arbitrary jumps. Formally, this means that $\Pi$ is sparse. In such cases, a few assets will be able to implement arbitrary feasible consumption plans since at any point in time a few assets can span the short-run uncertainty. Even though the examples will be nonrobust to changes in the transition matrix, they probably correspond better with standard intuition than the analysis above.

\subsection{An Example of Trading}

Both of our examples assume $H=2$ agents with CRRA Bernoulli-functions, where type one agents have relative risk aversion of $\gamma_{1}=0.5$ and type two agents have $\gamma_{2}=4$. The common discount factor equals $\beta=0.95$.

Our first example assumes $S=5$ exogenous states, with a Markov transition 
matrix

$$
\Pi=\left[\begin{array}{ccccc}
0.5 & 0.5 & 0 & 0 & 0 \\
0.5 & 0 & 0.5 & 0 & 0 \\
0 & 0.5 & 0 & 0.5 & 0 \\
0 & 0 & 0.5 & 0 & 0.5 \\
0 & 0 & 0 & 0.5 & 0.5
\end{array}\right]
$$

The first asset is stock with dividends $d^{s}=(1,1.5,2,2.5,3)^{T}$. This example approximates a dividend following a random walk except for the reflecting barriers when dividends are at their highest and lowest possible values.

The stock is in unit net supply and initial endowments are equal, $\theta_{-1}^{1 s}=\theta_{-1}^{2 s}=0.5$. The second asset is a riskless short-lived bond paying 1 unit of the consumption good in every state, $d_{y}^{b}=1$ for $y \in\{1, \ldots, 5\}$. Both agents have zero endowments of the bond, and they have no personal endowment of the consumption good; so $e_{y}^{1}=e_{y}^{2}=0$ for $y \in\{1, \ldots, 5\}$.

The algorithm of Section 3 can be used to compute equilibria even when markets are incomplete. The key condition is that the assets are able to implement the equilibrium consumption process through dynamic trading. This will be true here with our stock and bond since there are only two possible future states at any time, and the stock and bond can never have identical returns. Our algorithm tells us that $\lambda^{2}=0.982352$ and that the state-contingent consumption allocations are

$$
\begin{aligned}
& c^{1}=(0.191,0.572,1.004,1.457,1.920) \\
& c^{2}=(0.809,0.928,0.996,1.043,1.080)
\end{aligned}
$$

Type one agents have more volatile consumption than the more risk averse type two agents. When dividends are low, type one agents consume much less than type two agents, but this is reversed in the high dividend states. This kind of reversal is expected since each type begins at time zero owning exactly half of all wealth.

The state-contingent asset prices of the stock and the bond are

$$
\begin{aligned}
& q^{s}=(17.49,30.23,39.93,48.10,55.27) \\
& q^{b}=(0.749,1.181,1.024,0.986,1.020)
\end{aligned}
$$

The individual portfolios now vary across states. This does not contradict Theorem 2 since Theorem 2 is a generic result. The fact that the payoff matrix $\Pi$ in this example has many zero entries is a nongeneric property and is obviously critical here. The fact that each state has only two possible successor states implies that it is possible for two assets to span the space of returns in the short-run, and that trading in those two assets may be able to span all possible consumption plans. In this example, the asset prices and returns all line up so that this is true.

Let the end-of-period holding of stocks (bonds) by agent $h$ in state $j$ be denoted by $\theta_{j}^{h s}\left(\theta_{j}^{h b}\right)$. The consumption patterns, the asset prices, and the state-contingent 
budget constraints imply that the state-contingent end-of-period portfolio of type one agents is

$$
\begin{aligned}
& \theta^{1 s}=(0.464,0.502,0.578,0.604,0.600) \\
& \theta^{1 b}=(-1.090,-1.797,-4.700,-6.085,-5.846)
\end{aligned}
$$

The pattern of trading is interesting, illustrating a variety of factors. When dividends are high, the stock price is high, and the risk-tolerant type one agents hold the majority of the equity and are short in the bond market. Type one agents have high consumption and high net wealth in those states. This large holding of equity is necessary to finance type one consumption. As dividends fall type one agents unload their equity in order to finance consumption and pay off their debt. At the lowest dividend state, type two agents own the majority of the equity despite the fact that they are much more risk averse.

We should also note that this example is not a knife-edge case. In particular, a small change in any utility parameter or dividend parameter in this example will also produce a determinate equilibrium with trading. This holds since the critical matrices are nonsingular. Therefore, within the space of dividend processes with the zero pattern of this example, we have produced a robust example of trading.

\subsection{Example with Changing Variance}

The last example has a simple random walk character with reflecting barriers. The mean and variance of returns changes over time, making it difficult to explain the asset movements. One simple conjecture is that changes in variance will induce trade with the more risk averse agent selling some of his holdings to the more risk tolerant agent. The next example examines this conjecture.

We assume that same utility functions for type 1 and 2 trades, but change the transition matrix and the stock dividends in the previous example to create an example where the mean future dividend is fixed but the variance changes. We assume the Markov transition matrix

$$
\Pi=\left[\begin{array}{cccc}
0 & 0.5 & 0.5 & 0 \\
0.5 & 0 & 0 & 0.5 \\
0 & 0.5 & 0.5 & 0 \\
0.5 & 0 & 0 & 0.5
\end{array}\right]
$$

and assume that the new stock dividend process is $d^{s}=(1,2,4,5)^{T}$. In this example the time $t$ expectation of the time $t+1$ dividend is always 3 , but the variance of this expectation can change across states. In states 1 and 3 the dividend in the next period is either 2 or 4 , but is either 1 or 5 in states 2 and 4 . If trading was induced by changes in variance, then we might expect the more risk tolerant type 1 agents to buy stock from type 2 agents after a transition from state 1 to 2 or from state 3 to 2 . 
The asset prices of the stock and the bond are

$$
\begin{aligned}
& q^{s}=(17.77,44.34,75.87,88.55) \\
& q^{b}=(0.3030,1.4146,1.2935,2.8248)
\end{aligned}
$$

the portfolio policy of agent 1 is

$$
\begin{aligned}
& \theta^{1 s}=(0.5527,0.5315,0.5527,0.5315) \\
& \theta^{1 b}=(-1.9462,-0.5912,-1.9462,-0.5912),
\end{aligned}
$$

and the state-contingent consumption is

$$
\begin{aligned}
& c^{1}=(.1527, .9370,2.7821,3.7363) \\
& c^{2}=(.8473,1.0630,1.2179,1.2637) .
\end{aligned}
$$

The volatility of asset prices changes substantially as states change. In state one, the asset price is low, but the price in the next period is either (approximately) 44 or 76, but in state two, the price is 44 with future price equally either 18 or 89 . Similarly, expected price volatility is much higher in state four than in state two. Now the asset positions are identical in states 1 and 3 (2 and 4) since states 1 and 3 (2 and 4) have the same variance in returns.

The asset holding patterns are somewhat puzzling. The relatively risk-tolerant type one investors hold less equity in the riskier states 2 and 4 . When the dividend process moves from state 1 to state 2, dividend and price variance increases and type one investors sell shares to type two agents who are more risk averse, and they reduce their leverage. Consider also the situation in state two. If the next period's state is (apparently less risky) state one, then type one agents increase their equity holdings. This seems odd from a portfolio rebalancing perspective. However, it is not surprising when we examine the consumption pattern. When the dividend process moves from state two to state one, type one consumption plummets from 0.9370 to 0.1527, leading type one agents to save and increase their asset holdings.

These two examples highlight some important points. First, trading will occur when there are fewer assets than the total number of states. This is a reasonable assumption to make, even though it is a nongeneric condition in the theory. Second, portfolio trading patterns do not follow any simple rules. The equilibrium portfolios are determined by a variety of factors with riskiness of the assets being only one of them. Third, even in the cases where trading does occur, it is small in magnitude. Our examples encompass a wide variation in dividends and asset prices, and have agents with substantially different risk aversion. Still, the volume of trade is rather small. 


\section{Conclusions}

Analyses of asset markets often ignore volume. We show that standard intuition about volume is misleading. In particular, in generic dynamically complete markets of Lucas-style asset models, there will be no trading motivated by portfolio rebalancing considerations. This follows directly from the recursivity properties of any Pareto efficient allocation and the spanning properties of assets in a dynamically complete market.

This result implies that the reasons for trade lie in other considerations, such as life-cycle factors and asymmetric information (factors noted elsewhere), as well as incompleteness of the asset market. We display one kind of model with asset incompleteness which will generate trade for portfolio rebalancing considerations. The key property of these examples is that if the dividend process can be modelled by a Markov process with a sparse transition matrix, then a few assets can dynamically span all feasible consumption processes but only with some trading since the character of each asset changes as the dividend process evolves. While this later model is formally nongeneric, it may be a more realistic model of actual markets.

All of our results indicate that volume is not pinned down by simple rules such as the risk-tolerant buying up risky assets when they become riskier. In fact, the opposite happens in our sparse transition matrix examples. Pareto efficiency of the consumption process apparently has little to say about asset volume. In general, volume is sensitive to details of a market which are difficult to know. The fact that portfolio rebalancing alone will not produce substantial trading adds to the mystery of why asset markets are so active. 


\section{References}

[1] Arrow, K., "The Role of Securities in the Optimal Allocation of Risk-bearing," Review of Economic Studies 31 (1964), 91-96.

[2] Bewley, T. "Existence of Equilibria in Economies with Infinitely Many Commodities," Journal of Economic Theory 43 (1972), 514-540.

[3] Brock, W.A., and B. LeBaron, "A Dynamic Structural Model for Stock Return Volatility and Trading Volume," Review of Economics and Statistics, 78 (1996), 94-110.

[4] Debreu, G., Theory of Value, Yale University Press, New Haven (1959).

[5] Duffie, D., J. Geanakoplos, A. Mas-Colell, and A. McLennan. "Stationary Markov Equilibria," Econometrica 62 (1994), 745-781.

[6] Grossman, S. J. "Dynamic Asset Allocation and the Informational Efficiency of Markets," Journal of Finance 50 (1985), 773-789.

[7] Magill, M. and W. Shafer. "Characterization of Generically Complete Real Asset Structures," Journal of Mathematical Economics 19 (1990), 167-194.

[8] Judd, K.L., F. Kubler, and K. Schmedders. "Computing Equilibria in Infinite Horizon Finance Economies: The Case of One Asset," forthcoming in Journal of Economic Dynamics and Control (2000).

[9] Judd, K.L., F. Kubler, and K. Schmedders. "A Solution Method for Incomplete Asset Markets with Heterogeneous Agents," working paper (1999).

[10] Kreps, D. "Multi-Period Securities and the Efficient Allocation of Risk: A Comment on the Black-Scholes Option Pricing Model," in J. McCall(ed.): The Economics of Information and Uncertainty, The University of Chicago Press (1982).

[11] Lucas, R.E., Jr. "Asset Prices in an Exchange Economy," Econometrica 46 (1978), 1429-1445.

[12] Wang, J. "A Model of Competitive Stock Trading Volume," Journal of Political Economy 102 (1994), 127-168. 